\title{
Human Integration of Motion and Texture Information in Visual Slant Estimation
}

\author{
Albena Tchamova $^{1}$, Jean Dezert ${ }^{2}$, Nadejda Bocheva ${ }^{3}$, Pavlina Konstantinova ${ }^{1}$ \\ 1. Institute for Information and Communication Technologies, Bulgarian Academy of Sciences, \\ "Acad. G.Bonchev" Str., bl.25A, 1113 Sofia, Bulgaria, \\ Email: tchamova@bas.bg,pavlina@bas.bg \\ 2. ONERA - The French Aerospace Lab, F-91761 Palaiseau, France, \\ Email: jean.dezert@onera.fr \\ 3. Institute of Neurobiology, Bulgarian Academy of Sciences, \\ "Acad. G.Bonchev" Str., bl.23, 1113 Sofia, Bulgaria, \\ Email: nbbocheva@hotmail.com
}

\begin{abstract}
The present research is aimed to: (i) characterize the ability of human visual system to define the objects' slant on the base of combination of visual stimulus characteristics, that in general are uncertain and even conflicting. (ii) evaluate the influence of human age on visual cues assessment and processing; (iii) estimate the process of human visual cue integration based on the well known Normalized Conjunctive Consensus and Averaging fusion rules, as well on the base of more efficient probabilistic Proportional Conflict Redistribution rule no.5 defined within Dezert-Smarandache Theory for plausible and paradoxical reasoning. The impact of research is focused on the ability of these fusion rules to predict in adequate way the behavior of individuals, as well as age-contingent groups of individuals in visual cue integration process.
\end{abstract}

Keywords-Integration of visual stimulus characteristics; DSmT; probabilistic Proportional Redistribution rule no.5; Normalized Conjunctive rule; Averaging rule.

\section{INTRODUCTION}

The visual information about the $3 \mathrm{D}$ world utilized by humans is provided by a set of $2 \mathrm{D}$ images on the eye retina. It leads to uncertainty and/or discrepancy in image interpretations because the same projections could belong to different 3D objects. As an additional complexity, the visual system has to recover the information about objects' depth (i.e. the mutual disposition of objects) with respect to the observer. To overcome these difficulties one needs to utilize and combine in an effective way a variety of visual characteristics (or so called cues) in order to achieve inferences, more informative and potentially more accurate than if they were obtained by means of a single cue. The process of combining, manipulating and interpreting information in stimulus integration problem is beneficial because it allows the human visual system to estimate and perceive more accurately the objects' properties and to take appropriate actions, leading to improved reasoning (judgment) under uncertainty or/and possible conflicts between different visual stimulus. The uncertainty, associated with the utilized visual cues and the possible conflicts between

This paper is partially supported by contract D002-240/18.12.2008 with Bulgarian National Science Fund. them influences the decision making and action control in the process of human aging due to the increased level of internal noise in the visual system [1]. If the visual system neglects some of the available information [2], the visual signal/noise ratio will additionally deteriorate. Throughout the life cycle many aspects of vision and visual information processing decline and affect everyday task performance. 3D shape of objects and their spatial layout are specified on the base of both: static and dynamic visual cues. Age-related impairments in visual processing and perception are observed for both of them [3]. Therefore the task of vision inherently requires the integration of all available visual cue information to determine 3D object's shape. This paper focuses on human way of integration of motion and texture information in the process of object's slant estimation. Our goal is to reveal not only the age-related changes in the process of visual information assessment, but also the plasticity of the visual system to best adapt to these changes and to efficiently exploit all the available information in the visual scene in order to provide the visual system with a meaningful output, concerning more accurate and robust spatial information about the 3D objects. We will present and compare the performance of three fusion rules to model human way of visual cue integration: Normalized Conjunctive Consensus (NCC), Averaging (AVE), and the probabilistic Proportional Conflict Redistribution rule no.5 (pPCR5) defined recently within Dezert-Smarandache Theory (DSmT) for plausible and paradoxical reasoning. In section II we present briefly the visual cue integration problem and recall the principles of NCC, AVE and pPCR5 fusion rules. In section III, we present the experimental strategy and procedure, methods, subjects, involved in the experiments, stimulus and used apparatus. In section IV, the research reasoning logic is presented as well the results, obtained on the base of applied fusion rules. Concluding remarks are given in section V.

\section{Visual Cue Integration fOR Slant Estimation}

Vision provides a number of static and dynamic cues to the 3D layout of observed objects and scenes. Human show 
individual differences in their abilities to utilize these cues for judgments. The first source of visual information considered in this paper relates to $2 \mathrm{D}$ texture variations in the projection of a slanted plane. The texture elements alter their form and the degree of shortening depends on their relative position in the plane and relative orientation to the observer - the shortening of element form and the texture density are highest in the direction of plane's tilt. The degree of texture variation depends on surface slant and it is biggest in the most distant plane areas with respect to the observer. Another source of visual information considers the object's motion relative to the observer. The gradient of velocity in two orthogonal directions contains information about the object's slant and tilt. When both static and motion information is available, the efficient way of combining data, provided by them, leads to more accurate and robust estimation of object's geometry and to better understanding and recognition of the surrounding scenes and objects. The common ideas for visual cue combination in order to specify the viewer-dependent object's characteristics rely on the assumption of cue independence. There are various methods for modeling the visual cue integration process. Bayesian inference [4], [5] is a classical approach for modeling and processing probabilistic information. An ideal Bayesian observer was used to define the optimal weighting and combination of redundant visual cues [8], [9]. The main difficulties applying it concern the need of measurements' statistics and knowledge about the a priori information. The Bayesian framework was applied for modeling the spatial integration of auditory and visual information [6], for visual and haptic integration [7] where the main idea is that the human brain combines visual cues to obtain the most reliable estimate of the state of the world, i.e. the estimate in which the variance of the resulting combined cue is minimized. As it will be shown in our research, this kind of integration, being very sensitive to the sources with the bigger means, neglects part of available information, which is very unsatisfactory behavior in cases of combining conflicting visual cues. Generally visual data are not only inaccurate, incomplete and uncertain, but even conflicting, because the observer moves, or the surfaces could change their orientation in the particular scene, or one object occludes the other. All these data particularities must be incorporated in the process of human visual perception in order to provide a complete and accurate model of the real world and to improve the decision accuracy. In our study we will apply and compare the performance of three fusion rules: NCC rule, pPCR5, and AVE fusion rules to model the human process of visual cues integration.

\section{A. Normalized Conjunctive Consensus rule}

The Normalized Conjunctive Consensus (NCC) rule is used to combine simultaneously assumed independent visual cues. In case considered, the information obtained by the available visual cues is characterized by Gaussian likelihood functions with given means $\mu_{i}, i=1,2, .$. and standard deviations $\sigma_{i}, i=1,2, .$. , defining the uncertainty encountered in data. In case of two independent visual cues with one-dimensional
Gaussian distributions $p_{1}(x)=\frac{1}{\sigma_{1} \sqrt{2 \pi}} \exp -\frac{1}{2}\left(\frac{x-\mu_{1}}{\sigma_{1}}\right)^{2}$ and $p_{2}(x)=\frac{1}{\sigma_{2} \sqrt{2 \pi}} \exp -\frac{1}{2}\left(\frac{x-\mu_{2}}{\sigma_{2}}\right)^{2}$, the combined distribution based on NCC rule becomes:

$$
p_{N C C}(x)=\frac{1}{\sigma_{N C C} \sqrt{2 \pi}} \exp -\frac{1}{2}\left(\frac{x-\mu_{N C C}}{\sigma_{N C C}}\right)^{2}
$$

where $\quad \sigma_{N C C}^{2}=\frac{\sigma_{1}^{2} \sigma_{2}^{2}}{\sigma_{1}^{2}+\sigma_{2}^{2}} \quad$ and $\quad \mu_{N C C}=\sigma_{N C C}^{2}\left(\frac{\mu_{1}}{\sigma_{1}^{2}}+\frac{\mu_{2}}{\sigma_{2}^{2}}\right)$.

It is characterized with a mean, biased toward the function with the bigger of the two means, similar to Bayesian estimator. It is optimal (minimizes the variance of the error estimation), when the original distributions have close mean values. When two visual cues are in conflict, however, (characterized with distant distributions), NCC rule leads to neglecting (not utilizing) part of the available information, because the source with the bigger mean is weighted more heavily. In this case it is reasonable to keep the original distributions in the fused probability density function until it is possible to make reliable decision. This has been done by pPCR5 fusion rule defined in DSmT.

\section{B. Probabilistic Proportional Conflict Redistribution rule no.5}

The general principle of all Proportional Conflict Redistribution rules [10], Vol.3 is to: 1) calculate the conjunctive consensus between sources of evidence (different visual cues) 2) calculate the total or partial conflicting masses; 3) redistribute the conflicting mass (total or partial) proportionally on non-empty sets involved in the model according to all integrity constraints. The recently proposed non-Bayesian probabilistic Proportional Conflict Redistribution rule no.5 [11] is based on the discrete Proportional Conflict Redistribution rule no.5 (PCR5) [10], Vol.3, for combining discrete basic belief assignments. For completeness, we will discuss in brief the main idea behind the discrete PCR5. It comes from the necessity to deal with both uncertain and conflicting information, transferring partial or total conflicting masses proportionally only to non-empty sets involved in the particular conflict and proportionally to their individual masses. Basic belief assignment (bba) represents the knowledge, provided by particular source of information about its belief in the true state of the problem under consideration. Given a frame of hypotheses $\Theta=\left\{\theta_{1}, \ldots, \theta_{n}\right\}$, and the so called power set $2^{\Theta}=\left\{\emptyset, \theta_{1}, \ldots, \theta_{n}, \theta_{1} \cup \theta_{2}, \ldots, \theta_{1} \cup \theta_{2} \cup \ldots \cup \theta_{n}\right\}$, on which the combination is defined, the general basic belief assignment is defined as a mapping $m_{s}():. 2^{\Theta} \rightarrow[0,1]$, associated with the given source of information s, such that: $m_{s}(\emptyset)=0$ and $\sum_{X \in 2^{\Theta}} m_{s}(X)=1$. The quantity $m_{s}(X)$ represents the mass of belief exactly committed to $X$. Under Shafer's model assumption of the frame $\Theta$ (requiring all the hypotheses to be exclusive and exhaustive), the PCR5 combination rule for only two sources of information is defined as: $m_{P C R 5}(\emptyset)=0$ 


$$
\begin{aligned}
& \text { and } \forall X \in 2^{\Theta} \backslash\{\emptyset\} \\
& \qquad \begin{aligned}
& m_{P C R 5}(X)=m_{12}(X)+ \\
& \sum_{\substack{Y \in 2^{\Theta} \backslash\{X\} \\
X \cap Y=\emptyset}}\left[\frac{m_{1}(X)^{2} m_{2}(Y)}{m_{1}(X)+m_{2}(Y)}+\frac{m_{2}(X)^{2} m_{1}(Y)}{m_{2}(X)+m_{1}(Y)}\right]
\end{aligned}
\end{aligned}
$$

All sets involved in the formula are in canonical form. The quantity $m_{12}(X)$ corresponds to the conjunctive consensus, i.e: $m_{12}(X)=\sum_{X_{1}, X_{2} \in 2^{\Theta}} m_{1}\left(X_{1}\right) m_{2}\left(X_{2}\right)$. All denominators are different from zero. If a denominator is zero, that fraction is discarded. No matter how big or small the conflicting mass is, PCR5 mathematically does a proper redistribution of the conflicting mass since PCR5 goes backwards on the tracks of the conjunctive rule and redistributes the partial conflicting masses only to the sets involved in the conflict and proportionally to their masses put in the conflict, considering the conjunctive normal form of the partial conflict. PCR5 is quasi-associative and preserves the neutral impact of the vacuous belief assignment. The probabilistic PCR5 is an extension of discrete PCR5 version to its continuous probabilistic counterpart. Basic belief assignment, involved in discrete PCR5 rule is extended to densities of probabilities of random variables. For two independent sources of information with given Gaussian distributions $p_{1}(x)$ and $p_{2}(x)$, the obtained combined result becomes [11]:

$$
\begin{aligned}
p_{p P C R 5}(x)=p_{1}(x) \int \frac{p_{1}(x) p_{2}(y)}{p_{1}(x)+p_{2}(y)} d y+ & \\
& p_{2}(x) \int \frac{p_{2}(x) p_{1}(y)}{p_{2}(x)+p_{1}(y)} d y
\end{aligned}
$$

The behavior of pPCR5 fusion rule in comparison to NCC rule (1) could be characterized by two cases below:

Case 1: both densities $p_{1}(x)$ and $p_{2}(x)$ are close (Fig.1case 1). The combined density acts as an amplifier of the information by reducing the variance. Here pPCR5 acts as NCC fusion rule.

Case 2: the densities $p_{1}(x)$ and $p_{2}(x)$ are distant (Fig.1-case 2 ). Then the combined density keeps both original densities (not merging both densities into only one unimodal Gaussian density as NCC rule does), avoiding to neglect a part of the available information.

This new (from a theoretical point of view) property is very interesting and it presents advantages for practical applications as it will be shown in our particular research. Application of

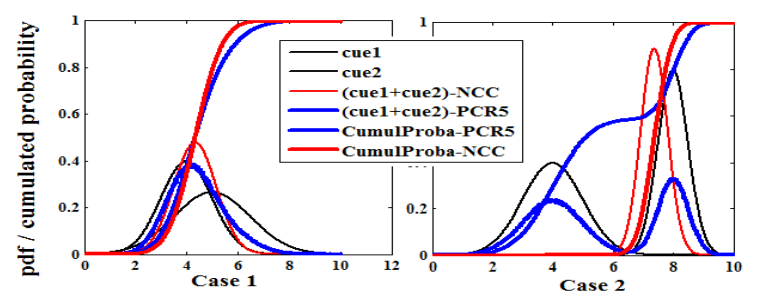

Fig. 1. Performance of pPCR5 fusion rule vs. NCC rule.

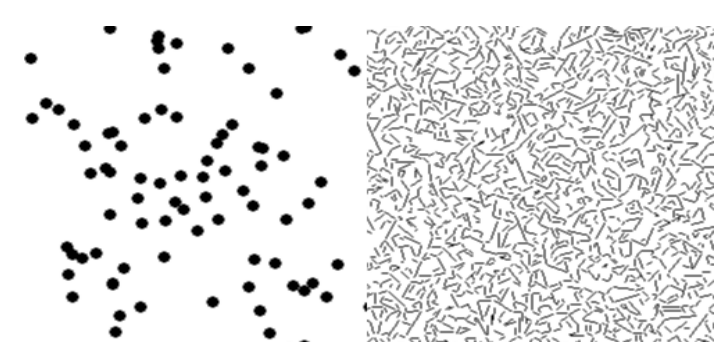

Fig. 2. Texture types: (left) dots, (right) lines.

pPCR5 fusion rule assures robustness to the potential errors and allows taking more reliable and adequate decisions in the process of integration of different cues in visual perception.

\section{Averaging rule}

The discrete simple Averaging rule consists in a simple arithmetic average of belief functions associated with sources of information (in our case particular visual cues). For given two sources of information defined with their discrete bba's: $m_{1}($.$) and m_{2}($.$) , for \forall X \in 2^{\Theta} \backslash\{\emptyset\}$, the combined distribution based on this rule becomes $m_{A V E}(X)=\frac{1}{2}\left(m_{1}(X)+\right.$ $\left.m_{2}(X)\right)$. This trade-off rule is commutative, but not associative. In case of two independent and equally reliable/trustful visual characteristics, associated with Gaussian distributions: $p_{1}(x)$ and $p_{2}(x)$, the combined distribution based on Averaging rule becomes:

$$
p_{A V E}(x)=\frac{1}{2}\left(p_{1}(x)+p_{2}(x)\right)
$$

\section{Experimental Goal, Methods, And Procedure}

The experimental goal is directed to: $(i)$ characterize the ability of human visual system to define the objects' slant on the base of only single cue available: Texture Information Only (referred as TIO case) or Motion Information Only (referred as MIO case), as well as in the case of both Texture and Motion information (referred as TM case), since human show significant individual differences in their abilities to combine and utilize both texture and motion information for judgments; (ii) evaluate the influence of human age on the assessment of objects' characteristics using available visual information.

\section{A. Observers}

Twelve elderly (mean age 74 years, range 67-85 years) and twelve younger (mean age 21 years, range 18-25 years) subjects took part in the experiments. All of them have passed eye examination. None of them reported having any major health problems.

\section{B. Stimuli}

The stimuli represent two slanted textured planes that form a symmetric horizontal dihedral angle. Two types of textures were rendered over the planes: dots (Fig.2-left) or a texture of non-intersecting lines (Fig.2-right).

Nine different sizes of the dihedral angles were used: 20 $\mathrm{deg}, 35 \mathrm{deg}, 50 \mathrm{deg}$, $65 \mathrm{deg}, 80 \mathrm{deg}, 95 \mathrm{deg}, 110 \mathrm{deg}, 125$ $\mathrm{deg}$, and $140 \mathrm{deg}$. To change the size of the dihedral angle, the 
slant of the two planes that hinged together was changed by an equal amount. One static and two dynamic conditions were generated. In all conditions the dihedral angle was presented in the middle of a computer screen under perspective projection and its vertical dimensions were fixed to 7 degrees of visual angle. In the static condition (TIO case) the information about the surface slant and consequently about the size of the dihedral angle is provided only by the changes in the texture over the planes. In the dynamic conditions (MIO case) the dihedral angle translated horizontally leftwards or rightwards with a speed of $6.4 \mathrm{deg}$ of arc/s. It changed direction on every $1.1 \mathrm{~s}$. In one of these conditions the texture specifies a flat object and thus, the information about the surface slant and the size of the dihedral angle is provided only by the motion. To achieve this, the texture coordinates were calculated relative to the eye coordinate system and they did not vary with the relative depth of the planes forming the dihedral angle. In the other dynamic condition (TM case) both the texture variation and the velocity of the object parts depend on the relative depth and therefore both specify the surface slant and the size of the dihedral angle.

\section{Apparatus}

The stimuli were presented on 21" Dell Trinitron monitor with Nvidia Quadro 900XGL graphic card. The monitor resolution was $1600 \times 1200$ pixels and the refresh rate was 85 $\mathrm{Hz}$. The stimuli were rendered on the screen using OpenGL. Grayscale images with 8 bit precision (256 colors) were used. The monitor was gamma-corrected using a lookup table.

\section{Experimental Procedure}

The observer sat in semi-illuminated room at a distance of $114 \mathrm{~cm}$ from the computer screen. The method of single stimuli was used. On every trial the observers had to compare the stimulus with an internal standard - a right dihedral angle. The task of the observers was to evaluate whether the presented dihedral angle was larger/smaller than a right angle. Each subject participated in 6 sessions. The sessions differed by the experimental condition and the texture type. The order of the experimental sessions was contra-balanced across observers. In every experimental session the 9 different values of the dihedral angle were presented in random order 30 times. Each experimental session started with a demo to familiarize the subjects with the texture types (Fig. 2) used in the study and the way the texture changes in the different experimental conditions. The proportion of responses "the dihedral angle is larger than the right angle" is estimated for all different experimental conditions and for each subject the resulting psychometric functions are obtained. For example the observed psychometric function, associated with the first tested young subject for the case TM is given in Table I.

All subjects passed a priori training session of 60 trials in which a particular checkerboard pattern (Fig.3-left) was used to texture the dihedral angle under perspective projection (Fig.3-right). It helps the subjects to get familiar with the task to perform. The results of training were not taken into account.

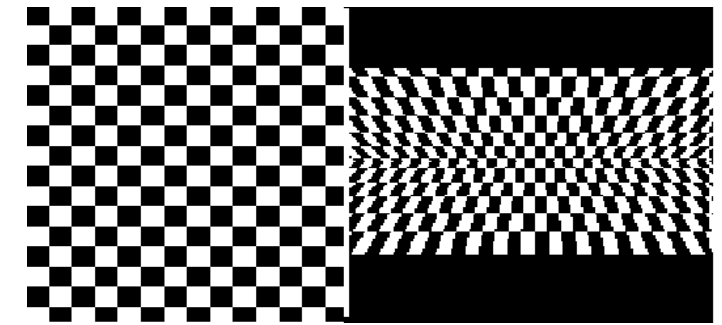

Fig. 3. Checkerboard pattern (left), Angle under perspective projection (right).

The dihedral angle remained visible on the screen until an answer was received. To give response the younger subjects used the buttons of a computer mouse while the elderly gave an oral response that was recorded by the experimenter.

\section{EXPERIMENTAL AND RESEARCH LOGIC}

Once having all psychometric functions, obtained for all different experimental conditions and for each subject in agecontingent groups, we should answer several questions:

Question 1. What is the effect of texture (dots, lines ) in MIO case? Does the manipulation of the texture we applied succeed to eliminate it's effect in MIO case?

Question 2. How human observers combine the visual cues in order to estimate surface's slant? Do they base their responses on a single cue (MIO or TIO) or on their combination TM? If a single cue is used, which one - TIO or MIO is more informative?

Question 3. What combination rule (NCC, pPCR5, or AVE) used to combine available visual cues predicts more adequately human's way of cue integration?

Question 4. What is the common trend, concerning the visual cue combination performance of both age-contingent groups, i.e the performance of the so-called averaged-people, associated with each group. We denote these trends as: trend of averaged-young-people and respectively trend of averagedold-people. They are based on combined individual behaviors in particular group, reveling its intrinsic behavior as a whole, reducing uncertainties associated with individual performances. All the tested subjects in age groups are considered as independent and equally reliable sources of information, because each subject provides his/her own psychometric function, associated with the TM combination process and should be taken into account in equal rights to derive these trends. Our goals are: $(i)$ to find out which combinational rule (NCC, pPCR5 or AVE) is able to model correctly and adequately such human age-contingent group trends in reasoning process; (ii) to analyze the special features, characterizing these trends.

TABLE I

EXAMPLE OF PSYCHOMETRIC FUNCTION.

\begin{tabular}{|c|c|c|c|c|c|c|c|c|c|}
\hline Angle's Value & 20 & 35 & 50 & 65 & 80 & 95 & 110 & 125 & 140 \\
\hline \hline $\begin{array}{c}\text { Answers } \\
\text { angle }>90 \text { deg } \\
\text { over } 30 \text { times }\end{array}$ & 0 & 0.12 & 0.17 & 0.73 & 0.9 & 0.95 & 0.98 & 1 & 1 \\
\hline
\end{tabular}




\section{RESUlts}

The experimental psychometric functions for both age groups and for all experimental conditions were compared using the pfcmp extension of MATLAB toolbox psignfit [14]. It implements a maximum-likelihood method [12] for fitting the psychometric functions and compares the parameters of the fits when estimated from the separate data sets and when the two sets are combined. As a result the significance value $p$ is produces as a measure of fit between the examined psychometric functions.

\section{- Results concerning Question 1 stated in Section IV.}

The results show that in MIO case the effect of the texture's type (line or dots) is effectively eliminated - for 10 out of 12 observers in each age group the null hypothesis of equal psychometric functions for both texture types could not be rejected at the assumed reliability level of $p=0.05$. For static TIO case the comparison of the psychometric functions obtained for line texture and for dots texture for both age groups, shows that the null hypothesis could not be rejected at $p=0.05$ for only 3 subject in each age group. These results suggest that the differences in the texture's type affect the subjects' performance significantly more in the static case. The smaller effect of the texture's types in MIO case provides indirect evidence that in these conditions the subjects' performance is based on the motion information.

\section{- Results concerning Question 2 stated in Section IV.}

In order to answer this question, we have analyzed and compared the experimental psychometric functions obtained for each subject in both age-contingent groups given the following cases:

- $\{$ dots-based TIO vs. dots-based MIO vs. dots-based TM $\}$

- $\{$ line-based TIO vs. line-based MIO vs. line-based TM

Older people rely more on the static information, especially in case of dots texture type. Five out of 12 subjects do not show significant difference $(p=0.05)$ in their performance for the TIO and TM case for dots texture, and 4 out of 12 subjects - for line texture. Young people rely more on the dynamic information: the psychometric functions for MIO and TM case do not differ significantly at $p=0.05$ for 5 out of 12 subjects for dots texture and 4 out of 12 - for line texture.

\section{- Results concerning Question 3 stated in Section IV.}

In order to answer correctly this question we should evaluate the performances of applied combinational rules in the process of visual cue integration to predict the model of human fusion performance on the base of theoretically predicted psychometric functions. A comparison between experimentally obtained and predicted psychometric functions for all tested cases is provided on the base of goodness-of-fit test [13], one important application of chi-squared criteria: $\chi^{2}=\sum_{j=1}^{J} \frac{\left(O_{j}-E_{j}\right)^{2}}{E_{j}}$ where $\chi^{2}$ is an index of the agreement between an ob$\operatorname{served}(O) /$ experimental and expected $(E) /$ predicted via particular fusion rule sample values of psychometric function. For our case $J=9$ represents the number of test angle values. The critical value of the test for $\nu=J-1=8$ degrees of freedom at assumed $p=0.01$ is $\chi^{2}=13.36$ [13]. This test is
TABLE II

CHI-SQUARED VALUES FOR OLDER SUBJECTS.

\begin{tabular}{|c|c|c|c|c|}
\hline Subject & dotsTM pPCR5 / AVE & dotsTM NCC & lineTM pPCR5 / AVE & lineTM NCC \\
\hline \hline 1 & $0.0653 / 0.0586$ & 0.1359 & $0.1775 / 0.2032$ & 0.5159 \\
2 & $0.8415 / 0.9015$ & 3.7232 & $0.0694 / 0.0663$ & 0.0796 \\
3 & $0.2359 / 0.2547$ & 0.4360 & $0.0827 / 0.0934$ & 0.1373 \\
4 & $0.6995 / 0.6876$ & 3.9117 & $0.1380 / 0.1461$ & 0.1522 \\
5 & $0.3618 / 0.3031$ & 0.3751 & $0.2982 / 0.3098$ & 0.3927 \\
6 & $0.1066 / 0.1304$ & 0.1387 & $0.1735 / 0.1943$ & 0.2261 \\
7 & $0.1859 / 0.1901$ & 0.1935 & $0.1881 / 0.2101$ & 0.4306 \\
8 & $1.6944 / 1.7958$ & 5.2330 & $0.3813 / 0.3114$ & 0.4585 \\
9 & $0.1697 / 0.2078$ & 0.8814 & $1.0045 / 1.0062$ & 1.5113 \\
10 & $0.0368 / 0.0561$ & 0.0566 & $0.1391 / 0.1411$ & 0.1519 \\
11 & $0.0909 / 0.0709$ & 0.1021 & $0.0577 / 0.0499$ & 0.0851 \\
12 & $0.2664 / 0.2564$ & 1.1320 & $0.1798 / 0.1682$ & 1.5873 \\
\hline
\end{tabular}

applied for both texture's types (dots and line) to the following pairs of psychometric functions:

- $\{$ MT case(experimental) - MT case (NCC rule) $\}$

- $\{$ MT case(experimental) - MT case (pPCR5 rule) $\}$

- $\{$ MT case(experimental) - MT case (AVE rule) $\}$

In general, the results show that the pPCR5 and AVE fusion rule predict more adequately than NCC rule human performance in all experimental cases. The differences between the experimental and estimated via pPCR5 and AVE rules psychometric functions for all observers in both age groups are smaller than those, obtained by NCC rule. For older subjects (Table II) all fusion rules predict psychometric functions that do not differ significantly from the experimental ones, but the differences in the fits are smaller in case of pPCR5 and AVE rules than in case of NCC rule application. For younger subjects (Table III), however, the NCC rule does not predict adequately the performance of the subjects in some conditions. For Subjects no. 5 and no. 6 (dots-based TM case) and for Subjects no. 4 and no. 9 (lines-based TM case) the obtained values (put in bold in Table III) significantly exceed the critical value of 13.36 . The graphical results reflecting younger subjects' no. 4 and no. 9 fusion behaviors in line TM case are shown in Fig. 4. These results reflects the situations, when the experimentally obtained psychometric functions, associated with single cues (TIO and MIO) are characterized with distant underlying Gaussian distributions. In this case pPCR5 and AVE fusion rules make predictions, which model more correctly and adequately human fusion behavior. They are almost similar, but pPCR5 rule performs better than AVE rule in these conflicting cases. In the integration process, based on NCC rule however, part of available information was neglected, because the visual cues with bigger means were weighted more heavily (as it was described in Section II A.).

\section{- Results concerning Question 4 stated in Section IV.}

In order to evaluate the common trend in the performance of both age groups, we started with the assumption that the tested subjects within each group are independent individual sources of information/answers and all of them are equally reliable. The results obtained for experimental and estimated (via different fusion rules) trends, concerning the visual cue combination groups' performance are presented in Fig. 5: subplots 1,3 for older group, and subplots 2, 4 for younger one. Subplots 1 and 2 show results for line texture's type and subplots 3 and 4 - for dots texture's type.

In order to compare the performance of different fu- 
TABLE III

CHI-SQUARED VALUES FOR YOUNGER SUBJECTS.

\begin{tabular}{|c|c|c|c|c|}
\hline Subject & dotsTM pPCR5 / AVE & dotsTM NCC & lineTM pPCR5 / AVE & lineTM NCC \\
\hline \hline 1 & $0.2976 / 0.3011$ & 0.8526 & $0.0218 / 0.0191$ & 0.0258 \\
2 & $0.0801 / 0.0932$ & 0.1456 & $0.1264 / 0.1525$ & 0.6591 \\
3 & $0.2182 / 0.2076$ & 0.2690 & $0.1157 / 0.1201$ & 0.1347 \\
4 & $1.4509 / 1.4432$ & 1.4716 & $0.6354 / 0.6523$ & $\mathbf{5 7 . 4 9 1 6}$ \\
5 & $8.1655 / 8.1762$ & $\mathbf{4 5 . 1 4 5 8}$ & $1.4695 / 1.4512$ & 2.4105 \\
6 & $3.2425 / 3.3195$ & $\mathbf{3 4 . 1 4 5 8}$ & $0.1953 / 0.2003$ & 12.2206 \\
7 & $0.0014 / 0.0021$ & 0.0079 & $0.2810 / 0.2957$ & 0.9054 \\
8 & $0.9201 / 0.8925$ & 6.6588 & $0.3542 / 0.3513$ & 0.9365 \\
9 & $0.4950 / 0.4861$ & 0.5160 & $0.8665 / 0.9341$ & $\mathbf{8 7 . 1 1 0 5}$ \\
10 & $0.7633 / 0.7527$ & 0.8304 & $0.1554 / 0.1599$ & 0.1927 \\
11 & $0.4202 / 0.4259$ & 0.4380 & $0.3949 / 0.3901$ & 0.3977 \\
12 & $0.6371 / 0.6458$ & 4.4540 & $0.0532 / 0.0525$ & 0.2447 \\
\hline
\end{tabular}

TABLE IV

CITY BLOCK ERRORS BETWEEN EXPERIMENTAL AND PREDICTED TRENDS.

\begin{tabular}{|c|c|c|c|}
\hline & PCR5 & NCC & AVErage \\
\hline \hline lineTM Older group & 0.03 & 0.10 & 0.04 \\
dotsTM Older group & 0.06 & 0.11 & 0.04 \\
\hline \hline lineTM Younger group & 0.02 & 0.12 & 0.04 \\
dotsTM Younger group & 0.02 & 0.11 & 0.03 \\
\hline
\end{tabular}

sion rules in estimating common trends' prediction the city-block errors between the corresponding pairs averagedyoung/old-people MT(experimental) - averaged-young/oldpeople (NCC/pPCR5/AVE rules) for both texture's types are given in Table IV. Results show ultimatively that experimentally obtained trends and those, based on pPCR5 and AVE fusion rules are very closed and for both age-contingent groups are two times less then those, obtained via NCC fusion rule. pPCR5 and AVE rules predict more correctly the human model of reasoning, than NCC rule. pPCR5 performs a little bit better than AVE rule, utilizing all the available information (TIO and MIO), even in case of conflict. NCC based trends are very sensitive to the sources (different subjects' psychometric functions) with the bigger means, neglecting that way part of the available information and acting as an amplifier of the information by reducing the variances.

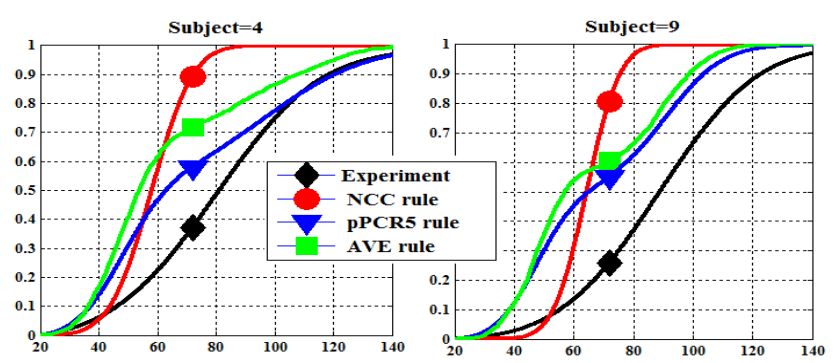

Fig. 4. Experimental and predicted performance for subject no.4 and no.9.

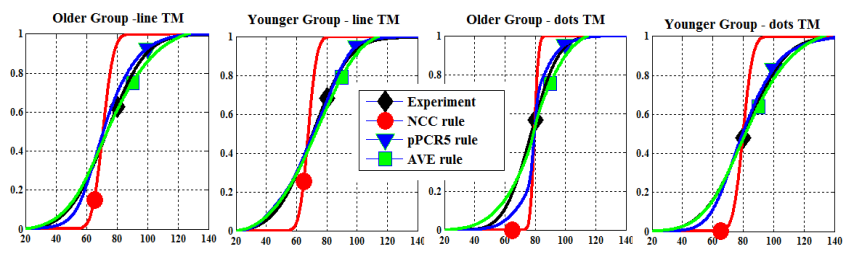

Fig. 5. Experimental and Predicted Trends in Performance of Age-related Groups.

\section{CONCLUSIONS}

The results obtained in this study show age-related difference in the performance of the subjects in estimating the threedimensional shape of the objects based on the texture and motion information. The task of the observers used in the study required the estimation of surface slant - a viewpoint dependent characteristic of the visual stimulation that is important for visual navigation and for object manipulation. Our data suggest that the younger people are more sensitive to differences in surface slant, but in the same time they are less accurate in their estimates. This cannot assure the robustness according to the potential errors during the experiments and leads to decisions which are less reliable than those taken by older people. Younger people as a group rely mainly on motion information neglecting the texture one. Elder people are characterized with less sensitivity to difference in the spatial characteristics of the three-dimensional objects in the real world, but they used to compensate this drawback by higher accuracy in their answers. Naturally this leads to ability to utilize correctly most of available stimulus information and then to improve the decision accuracy. The performance of both age groups in combining static and dynamic information is better described by the pPCR5 and AVE rule. In comparison to NCC rule, especially in conflicting cases pPCR5 fusion rules utilizes not only all available stimulus information, but this is achieved irrespective of the texture type (line or dots). That way pPCR5 fusion rule assures preserving the richness of stimulus data in the process of visual stimulus combination.

\section{REFERENCES}

[1] Pardhan, S. Contrast sensitivity loss with aging: sampling efficiency and equivalent noise at different spatial frequencies, JOSA A, Vol.21 (2), 2004, pp. 169-175.

[2] Falkenberg HK., Bex PJ. Sources of motion-sensitivity loss in glaucoma, Invest Ophthalmol Vis Sci., 48(6), 2007, pp. 2913-21.

[3] Owsley, C. Aging and vision, Vision Research, 2010.

[4] Bayes, T. An Essay towards solving a Problem in the Doctrine of Chances, Philosophical Transactions of the Royal Society of London, 1763, pp. 330-418.

[5] Sivia, D. Data Analysis, a Bayesian Tutorial, Clarendon (Oxford), 1996.

[6] Alais D., Burr D. The ventriloquist effect results from near optimal cross modal integration, Current Biology, 14, 2004, pp. 257-262.

[7] Ernst M., Banks M. Humans integrate visual and haptic information in a statistically optimal fashion, Nature, 415, 2002, pp. 429-433.

[8] Knill, D. Robust cue integration: A Bayesian model and evidence from cue-conflict studies with stereoscopic and figure cues to slant, Journal of Vision 7(7):5, 2007, pp. 1-24.

[9] Stocker A., Simoncelli E. Constraining a Bayesian Model of Human Visual Speed Perception, Advances in Neural Information Processing Systems, vol.17, 2005, pp. 1361-1368.

[10] Smarandache F., Dezert J. Advances and applications of DSmT for information fusion, Volumes 1, 2, 3, ARP, 2004-2009.

[11] Kirchner A., Dambreville F., Celeste F., Dezert J., Smarandache F Application of probabilistic PCR5 Fusion Rule for Multisensor Target, In Proc.of International Conference of Information Fusion, Qubec, Canada, July 9-12, 2007.

[12] Wichmann FA., Hill NJ. The psychometric function: I. Fitting, sampling and goodness-of-fit. Perception and Psychophysics 63(8), 2001, pp. 1293-1313. Perception and Psychophysics 63(8), 2001, 1314-1329.

[13] Matre, J., Gilbreath G. Statistics for Business and Economics, 3rd Edition, ISBN 0-256-03719-1, 1987.

[14] Psignifit software, http://bootstrap-software.org/psignifit/. 